\title{
1 Literarische Epistemologie
}

Für das postmoderne wissenschaftliche Wissen sind nach Lyotard zwei Tendenzen kennzeichnend: erstens das nahtlose Ineinandergreifen von Wissenschaft und Technologie ${ }^{1}$ als Folge der rasanten Entwicklung insbesondere der Informationstechnologie sowie zweitens die „Rückkehr des Narrativen in das NichtNarrative“ ${ }^{\text {2 }}$ als Folge „der inneren Erosion des Prinzips der Legitimität des Wissens“3 nach dem Zusammenbruch der „großen Erzählungen“. Lyotards Einschätzung dieser Entwicklung ist durchaus ambivalent: Zum einen sieht er die Gefahr, dass durch die wissenschaftlich-technische Evolution ein Homogenisierungs- und Uniformierungseffekt erzeugt wird, der auf sämtliche lebensweltliche und gesellschaftliche Prozesse übergreift und letztlich zur „Verallgemeinerung der binären Sprachen“, ${ }_{4}^{4}$ zur Reduktion von Erkenntnis auf das, was „in Informationsquantitäten übersetzt werden kann“, ${ }^{5}$ zur „Tilgung des Unterschieds zwischen hier/jetzt und dort/damals“, ${ }^{6}$ zum Verlust humaner Welt-, Geschichts- und Subjekterfahrung ${ }^{7}$ und damit zu einem umfassenden „Schwinden der Wirklichkeit“8 führen kann. Zum anderen, so Lyotard, legen gerade die Naturwissenschaften und die sie charakterisierende „Pluralität formaler und

1 Jean-François Lyotard mit J. Derrida, F. Burkhardt, G. Daghini u.a.: Immaterialität und Postmoderne, aus dem Französischen v. Marianne Karbe, Berlin 1985, S. 55-74, hier S. 58.

2 Jean-François Lyotard: Das postmoderne Wissen. Ein Bericht, hrsg. v. Peter Engelmann, Wien ${ }^{5} 2005$, S. 87.

3 Lyotard: Das postmoderne Wissen, S. 116.

4 Jean-François Lyotard: Eine Widerstandslinie [1984], in: ders.: Grabmal des Intellektuellen, hrsg. v. Peter Engelmann, Wien 1985, S. 53-67, hier S. 63.

5 Lyotard: Das postmoderne Wissen, S. 23.

6 Lyotard: Eine Widerstandslinie, S. 63.

7 Vgl. Jean-François Lyotard: Das Erhabene und die Avantgarde, in: Merkur 38 (1984), S. 151164, hier S. 160.

8 Jean-François Lyotard: Beantwortung der Frage: Was ist postmodern?, in: Postmoderne und Dekonstruktion. Texte französischer Philosophen der Gegenwart, mit einer Einführung hrsg. v. Peter Engelmann, Stuttgart 1990, S. 33-48, hier S. 42. Lyotards Befund, wonach mit der Moderne „eine Erschütterung des Glaubens und, gleichsam als Folge der Erfindung anderer Wirklichkeiten, die Entdeckung einher[geht], wie wenig wirklich die Wirklichkeit ist“ (ebd.), deckt sich mit der von Baudrillard angesichts des omnipräsenten Phänomens der Simulation getroffenen Feststellung vom „kybernetischen Stadium“ der Realität (Jean Baudrillard: Der symbolische Tausch und der Tod, München 1982, S. 117). Die Annihilierung von Realität beginnt freilich nicht erst dort, wo technische Instrumente, Apparate und Medien zum Einsatz kommen, sondern - und darauf hat eindringlich Heidegger hingewiesen - elementarer und universeller dort, wo Wahrnehmung, Denken und Handeln selbst bereits technologisiert sind. 
axiomatischer Systeme“9 ein alternatives Legitimationsmodell für den sozialen Alltag wie für die Paradigmen der Wissenschaft nahe, das „keineswegs das der besten Performanz ist, sondern der als Paralogie verstandenen Differenz“; ${ }^{10}$ dabei bezieht die Paralogie ihr Rechtfertigungspotential aus der Form der „kleinen Erzählung“, für die „imaginative Erfindung“, „offene Systematik, das Lokale, die Anti-Methode“ ebenso kennzeichnend sind wie die „Unvorhersehbarkeit von ,Entdeckung““ und ineins damit die „Bildung von Unsichtbarkeiten“, ${ }^{11}$ die Betonung des Dissens, die „Heteromorphie der Sprachspiele“ (lokal beschränkte Konsense und zeitweilige Verträge), ${ }^{12}$ die Selbstreferentialität des Diskurses und die Explikation metapräskriptiver Regeln ${ }^{13}$ bei gleichzeitigem Verzicht auf eine ,allgemeine Metasprache“. ${ }^{14}$

Mit dem wissenschaftstechnologisch bedingten „Schwinden der Wirklichkeit“15 gerät auch die Annahme von einer Äquivalenz zwischen Wissen und Wirklichkeit, Wissen und Wahrheit unter Generalverdacht. Dies umso mehr, als sich der naturwissenschaftliche Gegenstandsbereich seit der wissenschaftlichen

9 Lyotard: Das postmoderne Wissen, S. 128. Zum - gegenüber dem traditionell-deterministischen Wissenschaftsparadigma grundsätzlich verschiedenen - Legitimationspotential elementarer physikalischer und mathematischer Theorien der wissenschaftlichen Moderne und Postmoderne (Quantenmechanik, Relativitätstheorie, das Gödel'sche Theorem, die Thom'sche Katastrophentheorie, Mandelbrots Mathematik der Fraktale, Prigogines Theorie dissipativer Strukturen u.a.) vgl. ebd. S. 172-173: „Man gewinnt aus diesen [...] Forschungen die Idee, daß die Überlegenheit der stetigen, ableitbaren Funktion als Paradigma der Erkenntnis und Prognose im Verschwinden begriffen ist. In ihrem Interesse für die Unentscheidbarkeiten, die Grenzen der Präzisison der Kontrolle, die Quanten, die Konflikte mit nicht vollständiger Information, die ,Frakta', die Katastrophen und pragmatischen Paradoxa entwirft die postmoderne Wissenschaft die Theorie ihrer eigenen Evolution als diskontinuierlich, katastrophisch, nicht zu berichtigen, paradox. Sie verändert den Sinn des Wortes Wissen, und sie sagt, wie diese Veränderung stattfinden kann. Sie bringt nicht Bekanntes, sondern Unbekanntes hervor.“

10 Ebd., S. 173.

11 Ebd., S. 175-177.

12 Ebd., S. 191-192.

13 Die explizite „Immanenz des Diskurses über die Regeln, die seine Gültigkeit ausmachen“ ist nach Lyotard einer der auffallendsten Züge des postmodernen Wissens (ebd., S. 159). Unter metapräskriptiven Aussagen versteht Lyotard die Voraussetzungen, unter denen denotative Aussagen gemacht werden; sie „schreiben vor, welcher Art die Züge der Sprachspiele sein müssen, um annehmbar zu sein“, wobei die Akzeptanzforderung wiederum nur dadurch gerechtfertigt ist, „daß dies Ideen, das heißt neue Aussagen, hervorbringen wird“ (ebd., S. 187). Metapräskriptive Aussagen, so ließe sich sagen, zielen auf methodologische Transparenz wissenschaftlicher Behauptungen ab.

14 Ebd., S. 186.

15 Lyotard: Beantwortung der Frage: Was ist postmodern?, S. 43. 
Moderne zunehmend von der sinnlich-sichtbaren Natur zu einer sinnlich nicht mehr wahrnehmbaren, einer buchstäblich anästhetischen oder anaisthetischen ,Natur' verschoben hat, die sich der wissenschaftlichen Repräsentation entzieht und als undarstellbare nur mehr im Modus des Ästhetischen dargestellt werden kann: Die (wissenschaftstechnologische) Ästhetisierung von Natur und Wirklichkeit erscheint geradezu als die Kehrseite ihrer (wissenschaftstechnologisch bedingten) Anästhetisierung. ${ }^{16}$

In Del Giudices Roman Atlante occidentale (1985) und Michel Serres Lehrfabel Ablösung werden die von Lyotard beschriebenen Tendenzen postmoderner Wissenschaft thematisch. An ihnen gilt es nun weniger zu zeigen, dass und wie wissenschaftliche Erkenntnisse und Erkenntnisprozesse literarisch poetisiert und poetologisiert, sondern dass und wie die diesen wissenschaftlichen Erkenntnissen und Erkenntnisprozessen selbst immanenten Zusammenhänge von Anästhetisierung und Ästhetisierung ${ }^{17}$ poetisch und poetologisch reflektiert und expliziert werden. Das Hauptaugenmerk wird also auf die Frage nach den spezifisch epistemologischen Funktionen der Gattung poetica scientiae zu richten sein.

16 Einen ähnlich dialektischen ,Mechanismus“ beschreibt Heidegger, wenn er Technik auffasst als den „Verbrauch“ und die „Vernutzung des Seienden“ durch „die Rüstung im metaphysischen Sinne“, also dadurch, dass „der Mensch sich zum ,Herrn“ des ,Elementaren“ macht“ (Martin Heidegger: Überwindung der Metaphysik, in: ders.: Vorträge und Aufsätze, Pfullingen ${ }^{4} 1978$, S. 67-96, hier S. 87 f.). Damit bezeichnet Technik ein spezifisch menschliches Verhältnis und Verhalten gegenüber der Wirklichkeit, eine buchstäblich konsumierende Praxis, die die vorfindliche, differenzierte Wirklichkeit (einschließlich der Wirklichkeit des Menschen) tilgt gerade auch dort, wo diese technisch produziert wird. Andernorts kennzeichnet Heidegger den „Grundvorgang der Neuzeit“ explizit als die „Eroberung der Welt als Bild“ einerseits, als Abstraktion und Mathematisierung andererseits (Martin Heidegger: Die Zeit des Weltbildes [1938], in: Holzwege [1950], Frankfurt/M. 1980, S. 73-100, hier S. 76 u. 92).

17 „Ästhetisierung“ - und darauf wird immer wieder zurückzukommen sein - in der mehrfachen Bedeutung von: der sinnlichen Wahrnehmung zugänglich machen, Konstruktionen von Sichtbarmachung via medialer Techniken, Fabrikation von Wissen und Erkenntnis, aber auch kosmetische Eingriffe z.B. in wissenschaftliche Darstellungen des Undarstellbaren etc. 